\title{
Controversies in management of the bladder cuff at nephroureterectomy
}

\author{
Avery E. Braun, Abhishek Srivastava, Fenizia Maffucci, Alexander Kutikov \\ Division of Urologic Oncology, Department of Surgical Oncology, Fox Chase Cancer Center, Temple University Health System, Philadelphia, PA, \\ USA \\ Contributions: (I) Conception and design: AE Braun; (II) Administrative support: A Srivastava, A Kutikov; (III) Provision of study materials or patients: \\ All authors; (IV) Collection and assembly of data: AE Braun; (V) Data analysis and interpretation: All authors; (VI) Manuscript writing: All authors; \\ (VII) Final approval of manuscript: All authors. \\ Correspondence to: Avery E. Braun, DO. Division of Urologic Oncology, Fox Chase Cancer Center, Temple University Health System, 333 Cottman \\ Avenue, Philadelphia, PA 19111, USA. Email: braunave@einstein.edu.
}

\begin{abstract}
Upper tract urothelial carcinoma (UTUC) accounts for roughly $5 \%$ of urothelial carcinomas. Historically, the gold standard for high-risk or bulky low-risk UTUC was an open radical nephroureterectomy with formal bladder cuff excision (BCE). The development of novel endoscopic, laparoscopic, and robotic techniques has transformed this operation, yet no level I evidence exists at present that demonstrates the superiority of one strategy over another. While new approaches to nephroureterectomy in the last decade have shifted the management paradigm to decrease the morbidity of surgery, controversy continues to surround the approach to the distal ureter and bladder cuff. Debate continues within the urologic community over which surgical approach is best when managing UTUC and how various approaches impact clinical outcomes such as intravesical recurrence, recurrence-free survival (RFS) and disease-specific mortality (DSM). When focusing on the existing treatment algorithm, key metrics of quality include (I) removal of the entire specimen en bloc, (II) minimizing the risk of tumor and urine spillage, (III) R0 resection, and (IV) water-tight closure allowing for early use of prophylactic intravesical chemotherapy. In the absence of robust evidence demonstrating a single superior approach, the urologic surgeon should base decisions on technical comfort and each patient's particular clinical circumstance.
\end{abstract}

Keywords: Bladder cuff excision (BCE); nephroureterectomy

Submitted Dec 05, 2019. Accepted for publication Dec 25, 2019.

doi: $10.21037 /$ tau. 2020.01 .17

View this article at: http://dx.doi.org/10.21037/tau.2020.01.17

\section{Introduction}

Upper tract urothelial carcinoma (UTUC) is defined as urothelial carcinoma that affects the anatomic regions from the renal calyces and collecting systems down to the distal ureter. Albeit related to bladder cancer, UTUC is a distinct pathologic entity from an epidemiological, etiologic, and natural history perspective. UTUC comprises only $5-10 \%$ of cases of urothelial carcinoma, with approximately $5 \%$ localized to the lining in the kidney and $2 \%$ localized to the ureter $(1,2)$.

SEER data from 1975 to 2011 document a rise in prevalence and incidence of UTUC. A dramatic shift of patients presenting with non-muscle invasive disease occurred from $7.2 \%$ in 1973 to 1984 to $31 \%$ in 1994 to 2005 and ultimately $56 \%$ in 2005 to $2011(3,4)$. The remaining $44 \%$ of patients still present late in their clinical course with invasive, locally advanced, or metastatic UTUC $(5,6)$. Muscle-invasive UTUC has an abysmal prognosis with a 5 -year specific survival of less than $50 \%$ for pT2/T3 disease and less than $10 \%$ for pT4 disease $(7,8)$. Figure 1 illustrates the common diagnostic algorithm from workup, risk stratification to the management of UTUC.

The recent development of novel endoscopic, laparoscopic, and robotic techniques has transformed the 


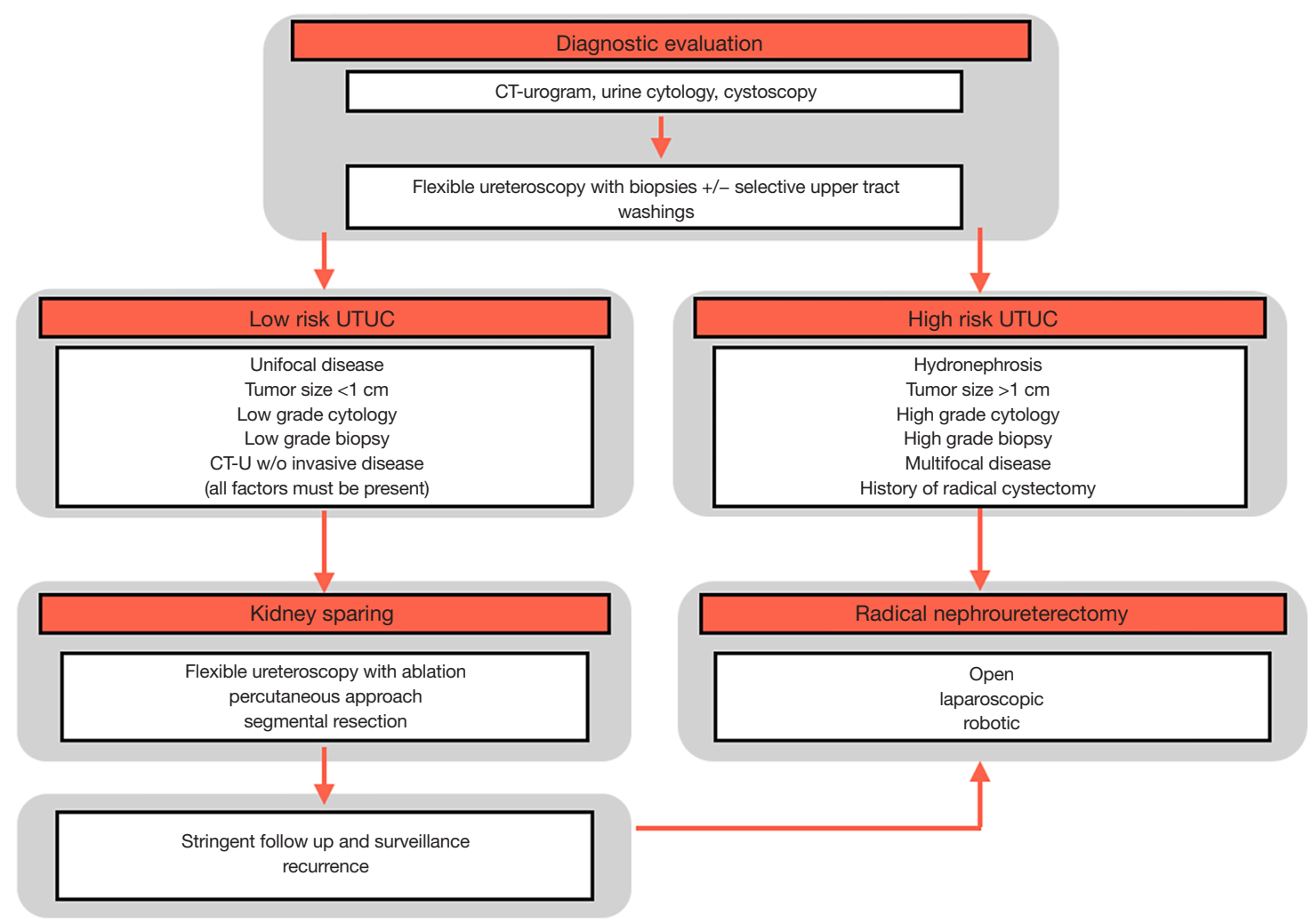

Figure 1 Common diagnostic algorithm from workup, risk stratification to the management of UTUC. UTUC, upper tract urothelial carcinoma.

surgical management of UTUC. Debate continues within the urologic community over which approach is superior and how various approaches impact clinical outcomes such as intravesical recurrence, recurrence-free survival (RFS) and disease-specific mortality (DSM). This paper will outline the current treatment paradigm with a specific focus on comparative techniques regarding nephroureterectomy with formal bladder cuff excision (BCE).

\section{Disease management}

The standard treatment of UTUC has undergone significant changes over the last decade (8). Improvements in radiographic imaging and endoscopic visualization of the entire urothelial lining have allowed clinicians to diagnose UTUC earlier and more accurately. The current gold standard for UTUC is radical nephroureterectomy (RNU) with BCE, which entails en bloc resection and removal of the kidney, ipsilateral ureter, and bladder cuff with the ureteral orifice. However, the strength of recommendation for the management of UTUC varies largely based on tumor location and risk $(9,10)$.

\section{Nephron-sparing approaches}

Historically, kidney-sparing treatment is a suitable treatment option over radical extirpative surgery in lowrisk patients. According to the European Association of Urology Guidelines on Upper Urinary Tract Urothelial Carcinoma 2017 Update, patients are stratified as low-risk UTUC when their disease is unifocal, $<1 \mathrm{~cm}$ in diameter, low grade on cytology and biopsy with no radiographic evidence of invasion (3). When all these factors are present, conservative management with nephron-sparing measure (NSM) is aimed at limiting morbidity without comprising oncological outcomes and kidney function; NSM can include counseling patients on options like observation, endoscopic ablation, and segmental ureterectomy. In select low-risk patients with low to moderate non-muscle invasive UTUC, there are several operative approaches that align with kidney-sparing objectives (11).

The least invasive technique available is ureteroscopy 
with endoscopic ablation using a flexible ureteroscope. This approach carries a Grade $\mathrm{C}$ recommendation from the EAU and is generally reserved for low-risk lesions (low grade, low volume, Ta) (3).

Segmental ureteral resection for ureteral tumors is a more invasive and controversial approach that aims to preserve the ipsilateral kidney. Resection with wide surgical margins allows practitioners to obtain adequate pathological specimens for accurate staging and grading. Ureteroureterostomy can be offered for patients with noninvasive, low-grade tumors of the proximal and mid ureter; however, risks of ischemic strictures are non-trivial (7). Furthermore, the segmental approach is not appropriate for distal ureteral tumors as complete distal ureterectomy with neocystotomy is the preferred surgical strategy in these cases $(2,7,8)$.

The use of UGN-101 (MitoGel ${ }^{\mathrm{TM}}$ ) for the treatment of upper tract disease has garnered significant interest (12). The Optimized DeLivery of Mitomycin for Primary UTUC Study or OLYMPUS Trial, an ongoing phase III clinical trial, assessed the clinical efficacy of mitomycin gel. Interim data suggest that $60 \%$ of patients achieve a complete response after induction therapy with $89 \%$ durability at 6 months (12). While UGN-101 is not currently FDA approved, the utility of this agent in the armamentarium for the management of low-grade UTUC continues to be closely investigated.

\section{Radical nephroureterectomy}

Nephroureterectomy can be divided into three critical operative steps: (I) nephrectomy, (II) ureteral extirpation, and (III) resection of the intravesical and intramural ureteral segment, known as BCE. The introduction of laparoscopic and robotic techniques has reshaped the standard approaches for all three steps, particularly BCE. Similar to debates regarding the trade-offs to open nephroureterectomy (ONU), laparoscopic nephroureterectomy (LNU), and robotic nephroureterectomy (RAL-NU), there is no single universally accepted technique for distal intravesical ureteral management during RNU, and strategy is largely dictated by surgeon comfort and preference.

ONU was the standard operative option for patients diagnosed with UTUC until the introduction and widespread adoption of minimally invasive surgical techniques. The surgical current approach to ONU varies and is dictated by patient factors like body habitus as well as physician factors like preference and experience. This variety in strategy is exemplified by the range of incisions utilized for ONU. As a multi-quadrant, trans-abdominal operation, the preferred approach to ONU is often a midline incision. However, some surgeons utilize a single thoraco-abdominal incision. Others prefer the two-incision approach where a flank or a subcostal incision is used for nephrectomy and proximal ureterectomy, while a Gibson, lower midline, or a Pfannenstiel incision is employed for resection of the distal ureter and bladder cuff.

LNU was first introduced and described by Clayman et al. in the 1990s. Since its introduction, it has largely replaced ONU in many high-volume institutions. The rationale behind the transition from ONU to LNU includes the demonstrated benefit of a minimally invasive approach, marked by low morbidity and expeditious convalescence (13). More specifically, patients who have undergone LNU have decreased blood loss, less post-operative pain, shorter length of hospitalization, and quicker time to recovery in comparison to ONU (13-15). In robust clinical studies, LNU has repeatedly shown comparable oncologic efficiency to ONU and has quickly achieved acceptance as an alternative treatment $(1,7,13,16)$. Over the last two decades, LNU has been utilized widely in the setting of renal pelvis and proximal ureteral tumors and has largely replaced ONU as the standard of care for UTUC that requires extirpation.

The robotic approach to nephroureterectomy has been increasingly adopted over the last decade since its introduction to this space in 2006 (17) as it may combat the challenge of addressing the BCE laparoscopically. With appropriate port placement, the availability of longer instruments, and maneuverability with the da Vinci ${ }^{\mathrm{TM}}$ system, RAL-NU has now become a feasible, albeit more costly, alternative to $\operatorname{LNU}(17,18)$.

\section{Bladder cuff}

After the kidney and ureter are freed from their respective surrounding structures, attention is turned towards the final portions of the operation, which include resection of a small length of the ureter $(<1 \mathrm{~cm})$ in the intramural portion of the bladder cuff and the ureteral orifice followed by cystotomy repair. In our recently published AUA News article, we noted that while $\mathrm{BCE}$ is a time-consuming and surgically challenging portion of the case, the temptation to leave the intramural segment behind has deleterious oncologic effects $(1,2)$. The oncologic importance of formal and complete $\mathrm{BCE}$ is underscored by the fact that the risk of tumor recurrence within this residual ureteric stump can 


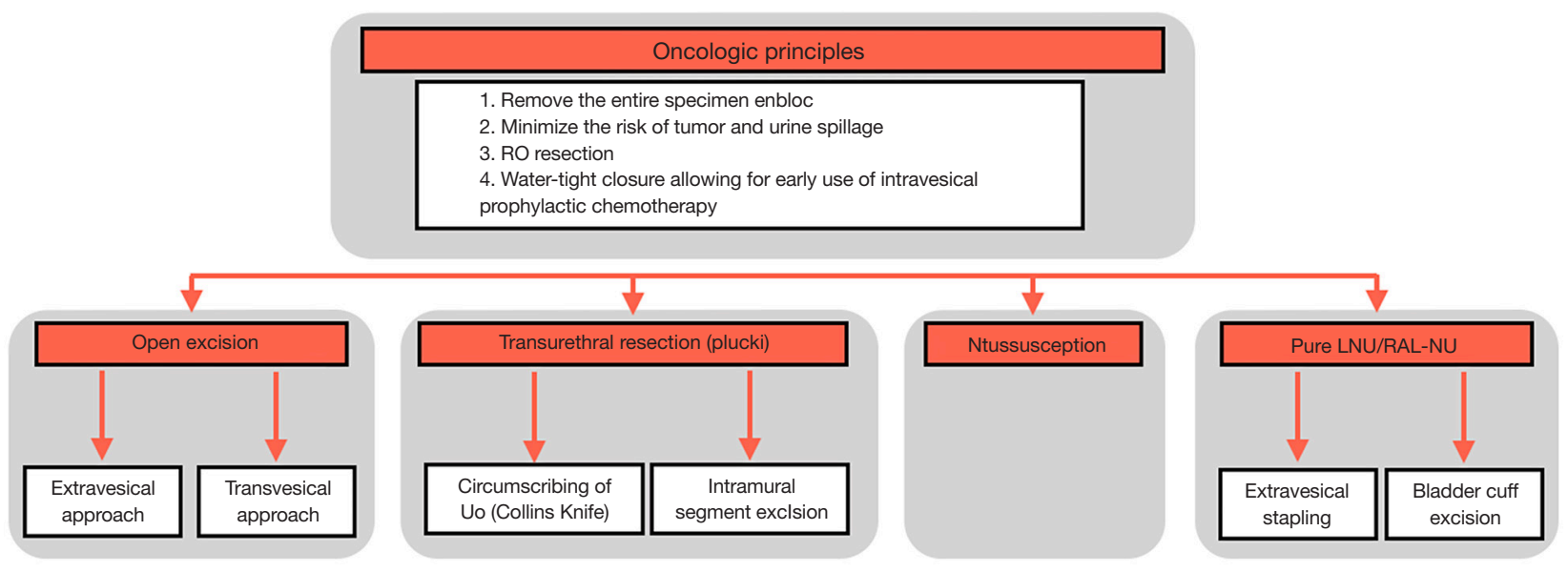

Figure 2 Management options pertaining to the management of the distal ureter and bladder cuff. UO, ureteral orifice.

be as high as $30-65 \%$ (19-21). Numerous publications have looked at the prognostic impact of BCE at RNU including a paper by Lughezzani et al. that concluded BCE must continue to be the standard of care and be performed at the time of nephroureterectomy (22).

Formal or definitive management of the distal ureter and bladder cuff can be accomplished via open, endoscopic, laparoscopic, or robotic approaches. The traditional bladder cuff management is excision, which can be done with an open extravesical or open transvesical approach. Alternative approaches include transurethral resection via Collins Knife (also known as the "pluck" technique), ureteric intussusception, pure laparoscopic extravesical excision, laparoscopic extravesical stapling, and pure RAL-NU $(1,2,16,19)$. Despite the growing number of multicenter, retrospective, comparative studies and case series published on the management of the bladder cuff, the most recent guidelines continue to acknowledge limitations and therefore support low-grade recommendations when it comes to BCE (3).

The choice in technique for the BCE is at the discretion of the surgeon and includes two key steps: (I) excision and (II) trigonal cystotomy closure.

The combination of intravesical ureteral excision and trigonal cystotomy closure strives to achieve the following surgical goals: (I) remove the entire specimen en bloc, (II) minimize the risk of tumor and urine spillage, (III) R0 resection, and (IV) water-tight closure allowing for early use of intravesical prophylactic chemotherapy.

Conforming to strict, well-establish oncological surgical principles is critical, and numerous retrospective studies have been published to evaluate each approach to the management of the bladder cuff at the time of nephroureterectomy $(1,2,7)$. Figure 2 illustrates options pertaining to the management of the distal ureter and bladder cuff.

\section{Open excision}

The open resection is the standard approach against which all other techniques are compared. It is typically performed after the nephroureterectomy portion of the procedure. The traditional open approach to the distal ureter and bladder cuff is open distal ureterectomy using an extravesical, transvesical, or combined approach. The open extravesical method occurs via a Gibson, Pfannenstiel, or lower midline incision with excision of a cuff of the bladder without cystotomy. This technique requires mobilization of the distal ureter to the point of the intramural ureter. The ureter is then placed on traction, aiding complete visualization of the intramural ureter prior to proceeding with ligation and division. Figure 3 illustrates the steps of formal open BCE as described (23).

Since extravesical dissection can be quite challenging in obese patients or those with thick bladders, anterior cystotomy is often preferable. Anterior cystotomy allows for the complete excision of the ureteral orifice and bladder cuff under direct visualization.

\section{Transvesical ligation of ureteral tunnel}

With the advent of laparoscopic surgery in the 1990s, 

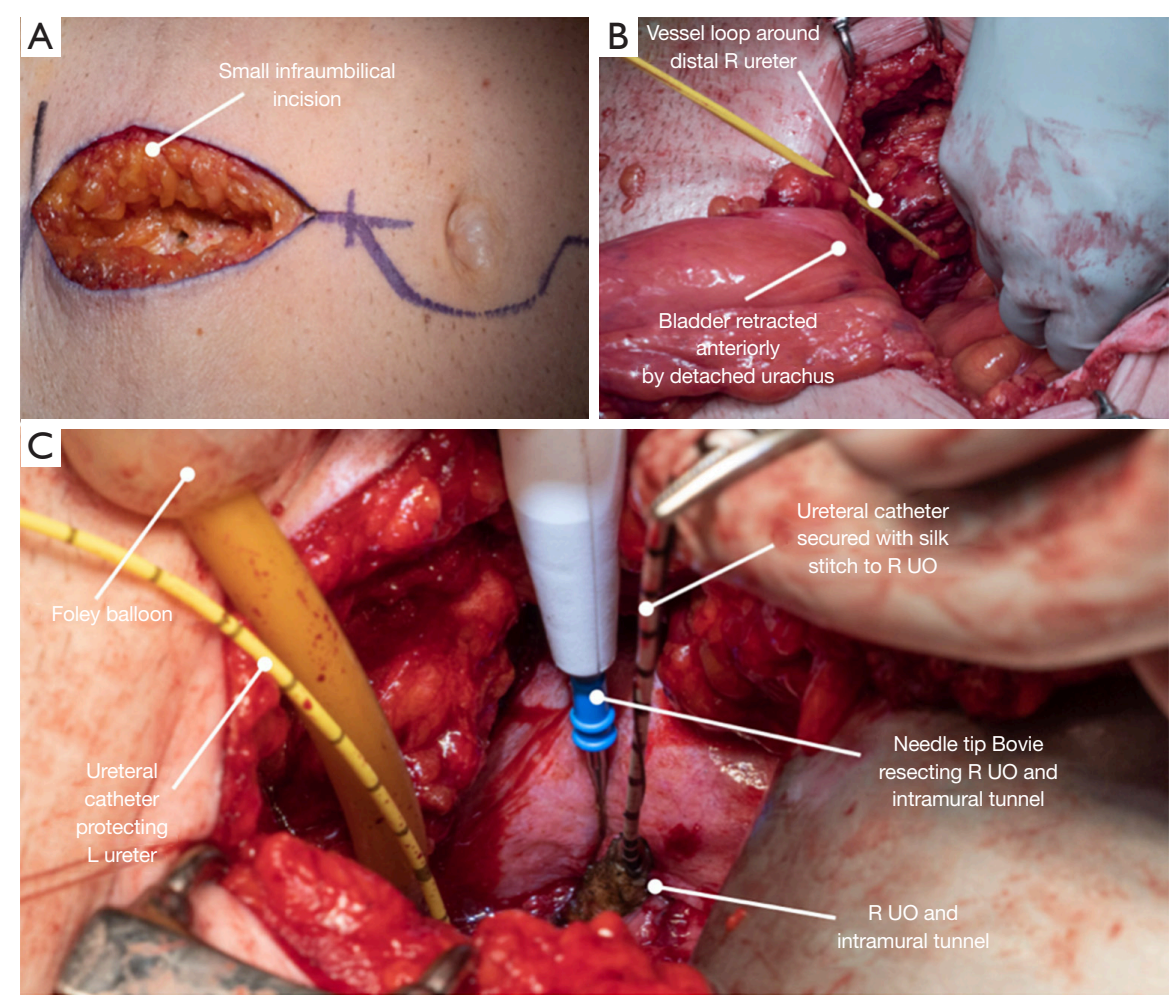

Figure 3 Steps of formal open BCE. (A) Small midline infraumbilical incision to perform formal open BCE. (B) Identification of the right ureteral orifice with retraction of the distal right ureter with vessel loop and bladder by detached urachus. (C) Open intravesical approach accomplished by cannulating right ureteral orifice with ureteral catheter and resecting ureteral orifice and bladder cuff with needle tip Bovie. Reproduced with permission by AUA (23).

novel approaches to BCE were introduced $(14,15)$. One of these techniques is transvesical ligation and detachment, which combines elements of endoscopy and laparoscopy. Execution of this approach involves filling the bladder to capacity via cystoscopy prior to placing one or two $5-\mathrm{mm}$ laparoscopic trocars transvesically. A laparoscopic loop is then placed around the ureteral orifice to occlude it from seeding prior to introducing a Collins Knife to excise the bladder cuff $(24,25)$.

\section{Intussusception technique}

The use of intussusception to address the bladder cuff at the time of RNU was first described by McDonald in 1953 but has undergone numerous modern modifications since its conception (25). To begin, a ureteral catheter is inserted into the distal ureter via a cystoscope. The distal ureter is then tied off extravesically and transected between the bladder cuff and the tie. Cystoscopic dissection of the bladder cuff is then performed with a Collins Knife. The transected distal ureter is secured to the ureteral catheter, and gentle traction on the catheter intussuscepts the ureter into the bladder. The ureter is then removed transurethrally. This technique has garnered skepticism given the theoretical risk of seeding the bladder urothelium with malignant ureteral mucosa cells at the time of intussusception, but Clayman et al. found it to be a safe and reliable technique with no evidence of local recurrence out to five years in their study (25).

\section{Transurethral ureteral tunnel management}

The endoscopic method for distal ureter and BCE arose as an alternative to obviate the low abdominal incision. The use of the "pluck" technique was first described in 1998 by Keeley and Tolley in a series of patients managed by LNU (26). It is accomplished either by utilizing a transurethral approach to resect the ureter in the line of its intramural segment with a resectoscope loop, or by circumscribing the ureteric orifice and intramural ureter using Collins knife. This approach is often referred to as 

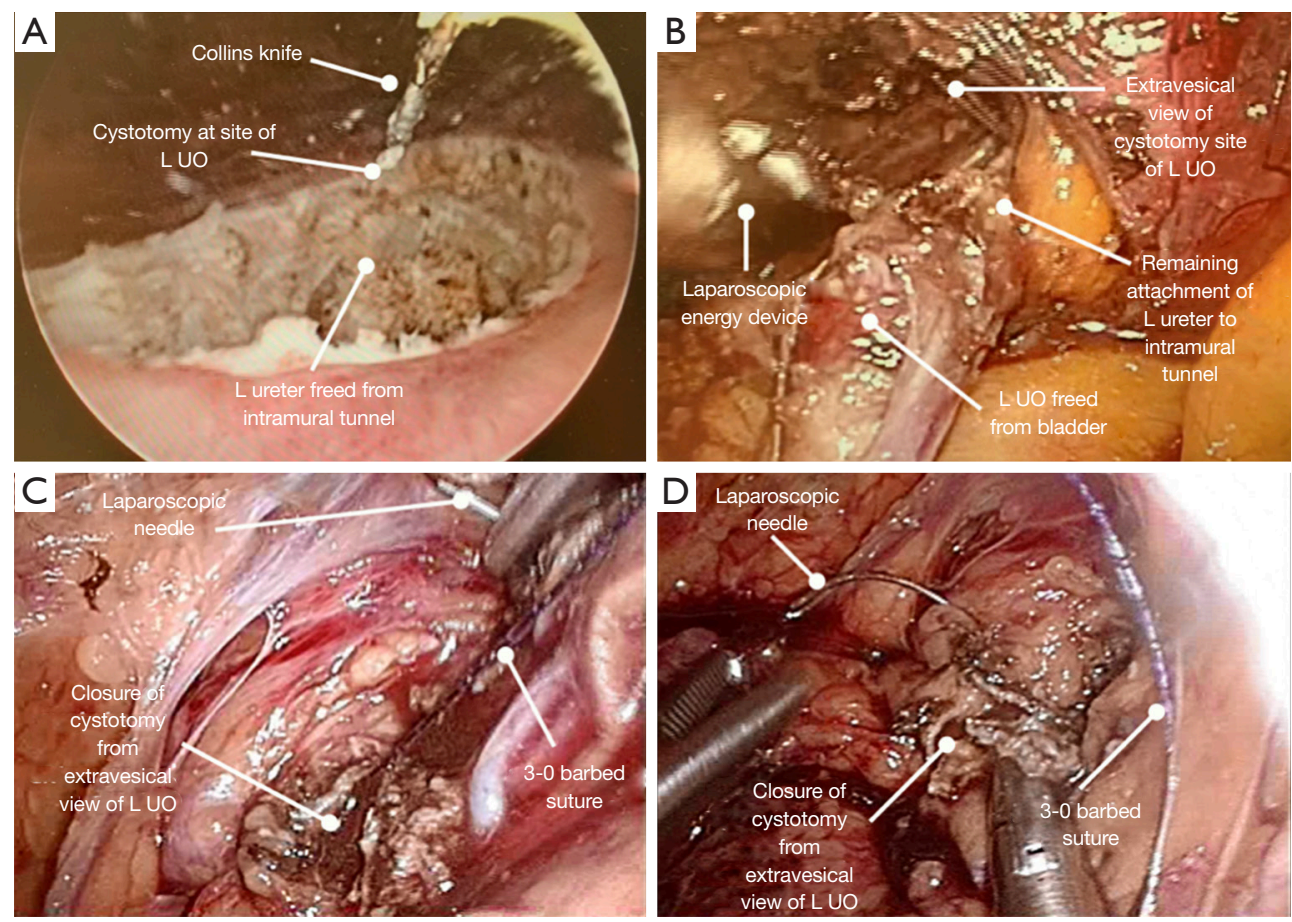

Figure 4 Steps of Collin's knife and laparoscopic approaches to BCE. (A) Endoscopic approach with use of Collins Knife to free distal ureter from bladder cuff at the cystotomy site. (B) Laparoscopic approach with use of energy device to free distal ureter from bladder cuff through an extravesical cystotomy. (C) Closure of the extravesical cystotomy at the ureteral orifice using 3-0 barbed suture. (D) Visualization of the laparoscopic needle at time of laparoscopic extravesical closure. Reproduced with permission by AUA (23).

the "pluck" technique as the ureteric orifice is resected deep into extravesical fat, allowing subsequent plucking of the entire ureter from above during the time of the nephrectomy. As such, the endoscopic portion is often performed prior to the laparoscopic or robotic nephrectomy $(16,20)$. Figure 4 depicts the combination of using the Collins knife and laparoscopic approaches to BCE (23).

This technique has sparked concerns among the urologic community with regards to potential local recurrence of the residual ureteral stump and urinary bladder tumor recurrence secondary to tumor seeding (27); however, no robust evidence supports these concerns. Over the years, several methods have been trialed to minimize tumor spillage, including pneumovescium with endoloop ligation, ureter clipping prior to an endoscopic approach, balloon occlusion of the distal ureter, coagulation of orifice, and fibrin sealant injection (28-31).

\section{Total LNU}

As surgeons shifted to LNU as the standard for UTUC treatment in the 1990s, a total laparoscopic approach became a more attractive option for management (24). Exclusive use of laparoscopic techniques for BCE management carries the attractive benefits of reduced operative time and avoidance of entry into the urinary tract system of the distal ureter. With a laparoscopic approach, dissection of the distal ureteral is performed extravesically and carried down to the intravesical level where detrusor muscle is split. Sequential retraction of the ureter in an antegrade fashion allows the orifice to be everted, providing access for a laparoscopic endovascular stapling device to ligate and divide at the specimen at the level of the bladder. While LNU is widely utilized, these techniques are challenging to master and require advanced laparoscopic skills.

\section{Total RAL-NU}

RAL-NU is now frequently utilized for UTUC as it lowers barriers to minimally invasive extirpation and potentiates suturing of the cystotomy $(17,24,32)$. A roboticassisted nephroureterectomy was first described in the 


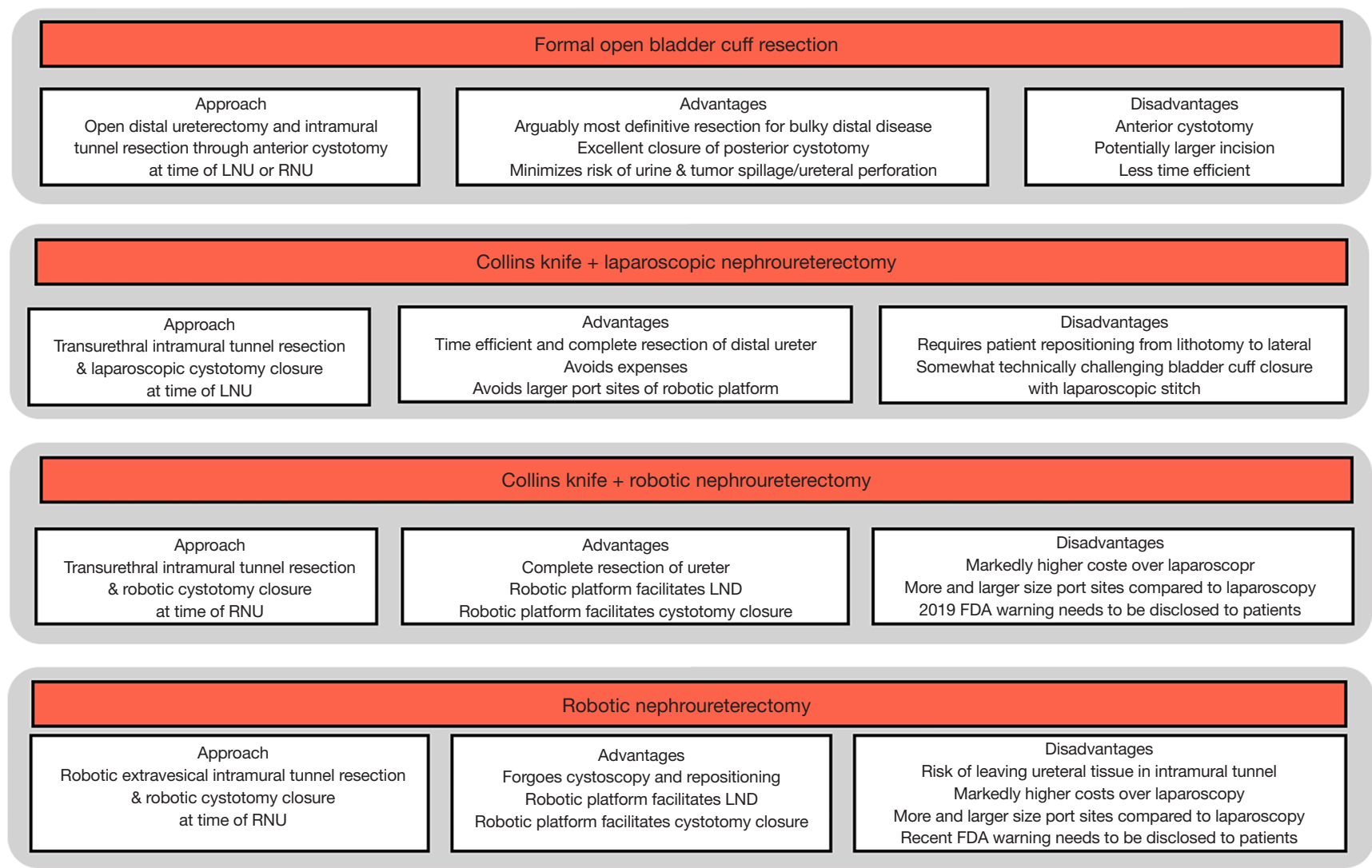

Figure 5 Various surgical options for radical nephroureterectomy with commonly cited advantages and disadvantages for each. LNU, laparoscopic nephroureterectomy; RNU, radical nephroureterectomy.

early 2000s by Gill et al. in which the distal ureterectomy was completed with an open BCE (14). That same year, Nanigian et al. reported the first use of the robotic excision for distal ureterectomy but performed the nephrectomy laparoscopically (32). Pure robotic-assisted LNU with BCE (RNU-BCE) was subsequently described by Eandi et al., who indicated repositioning of their patients and reorientating of the robot for deep pelvic dissection (33). This approach proved cumbersome, adding 30-50 minutes of operative time to re-dock, re-drape, and re-prep the patient for BCE. Lee et al. proposed a novel port configuration that made pure RNU-BCE possible without undocking (34). The advent of the $\mathrm{Xi}$ Da Vinci ${ }^{\mathrm{TM}}$ robotic system has afforded further improvements in maneuvering the robotic platform across the abdominal cavity $(32,33)$.

\section{Critical review of approaches to BCE}

RNU for treatment of UTUC aims to remove the entire ipsilateral urinary tract (kidney, ureter, bladder cuff, and ureteral orifice) in en bloc while avoiding tumor and minimizing urine spillage. Figure 5 lists the various surgical options during RNU with commonly cited advantages and disadvantages for each. Comparative effectiveness data between these various approaches (formal open bladder cuff resection, Collins Knife + LNU, Collins Knife + RAL$\mathrm{NU}$, and RAL-NU) are uniformly retrospective and often report contradicting results. For example, Li et al. report no differences in the local or distant recurrence rates, recurrencefree survival, or cancer-specific survival between the open, laparoscopic, and RAL-NU in a retrospective single-center study (35). In contrast, the UTUC Collaboration group published a multi-centered retrospective study by Xylinas et al. comparing the three different approaches and found the endoscopic approach was associated with a higher rate of intravesical recurrence compared to the transvesical and intravesical approaches (1). However, despite those findings, the overall and cancer-specific survival rates for each 
approach were the same $(1,36)$. Clearly, such data are limited by variations in case-mix and selection bias. In the absence of robust data, it is essential to consider the risks, trade-offs, and deliverables of each approach.

\section{ONU with Formal BCE}

As described previously, BCE excision during ONU can be approached two different ways: extravesically and transvesically. Walton et al. reviewed 372 patients who were treated with either extravesical excision of ureter and cystotomy encompassing the ipsilateral ureteral orifice or intravesical excision via a separate anterior cystotomy with circumferential excision of the distal ureter (36). At intermediate follow up of 47 months, investigators found there was no statistically significant difference in 5-year RFS between the two groups (36). Importantly, these two approaches demonstrated equivalent outcomes with regard to disease recurrence and cancer-specific mortality $(36,37)$.

Seisen et al. reached different conclusion in a 2015 systematic review of the literature to investigate factors influencing intravesical recurrence after treatment of UTUC with RNU (38). Cumulative analysis of 18 retrospective studies suggested significant patient, tumor, and treatment-specific risk factors that were associated with intravesical recurrence after ONU (38). The authors concluded that extravesical BCE was a risk factor for intravesical tumor recurrence (38). Furthermore, while locoregional recurrences after RNU are rare, a study by $\mathrm{Li}$ et al. reported slightly higher but not statistically significant local recurrence rates in patients undergoing open intravesical verses extravesical approaches (35).

Allard et al. compared open methods of BCE during LNU in a series of 110 preselected patients (15). With a median follow-up of 22 months, urothelial recurrences occurred in $26.7 \%$ and $38.1 \%$ of those who underwent extravesical and intravesical approaches, respectively (15). Rates of metastasis by BCE method were $20.6 \%$ and $10.0 \%$ for extravesical, and intravesical techniques, respectively (38). Ultimately, no statically significant difference was found between the groups in regard to the BCE method in association with freedom from recurrence and metastases $(15,19)$.

The location of the ureteral tumor may impact treatment outcomes. A systematic review of 42 studies comparing ONU and LNU suggested superior oncological outcomes with open BCE in patients with locally advanced, highrisk ureteral tumors (pT3/pT4), particularly with bulky distal ureteral masses $(10,39)$. While utilization of ligation/ clipping/stapling of the ipsilateral ureter in LNU minimizes proximal tumor spillage during BCE, open BCE through an infra-umbilical or Gibson incision potentially allows for more precise handling of the ureter and most reproducible and consistent control of tumor/urine spillage $(39,40)$.

\section{Endoscopic BCE}

The "pluck" method of transurethral incision of the bladder cuff with Collins Knife has been rigorously assessed for peri-operative and oncologic outcomes (10). One drawback to the "pluck" method when it was first being adopted was the need to reposition the patient from endoscopic lithotomy to supine for ONU or flank for LNU. However, numerous modified supine positions have been described in the literature since; Ou et al. used a "Johnnie walker position" with extended and abducted legs which has been adopted by many large volume institutions given its operative time-savings of roughly twenty minutes (decreased from 207 to 150 minutes in their series) (31).

One of the most significant drawbacks to the "pluck" technique was the theoretical concern for an increased risk of tumor spillage ergo increased retroperitoneal or bladder recurrence $(29,36)$. Studies suggest that the transurethral method should be avoided in patients with high-grade tumors or distal ureteral tumors near the bladder cuff (40); however, Rai et al. reported that the "pluck" method did not show a higher recurrence rate in those with high-grade or distal ureter tumors (41).

As a result of the proposed oncologic risk of the endoscopic approach, numerous modifications of the "pluck" technique have continually been published from Gill et al. in 1999 to Cormio et al. in 2013 (14,42). Some of these modifications included pneumovescium with Endoloop ligation, ureter clipping prior to an endoscopic approach, balloon occlusion of the distal ureter, coagulation of orifice, and fibrin sealant injection (31). Agarwal et al. in 2008 first described the use of the Endoloop instrument to occlude the ureteral orifice while circumscribing the bladder cuff transurethrally with Collins Knife (43). One of the later iterations by Cormio et al. described a technique where the ureter is endoscopically occluded with a $5 \mathrm{~F}$ ureteral catheter with a Fogarty balloon prior to transurethral resection of the bladder cuff (42). Ultimately, the necessity of these maneuvers is unclear with limited published data limited to short-term outcomes.

Luo et al. published retrospective outcomes of a Taiwanese cohort in 2014, showing no difference in the 
metastasis and cancer-specific mortality between the extravesical and endoscopic BCE groups (10).

Fragkoulis et al. compared ONU with open resection of the bladder cuff against transurethral resection of the intramural ureter (44). In this Greek cohort, investigators reported that total operative time was statistically shorter for transurethral resection; patients treated with open excision had a statistically significantly shorter duration of catheterization. From an oncologic perspective, they found no difference in bladder recurrence rate or CSS at five years. These conclusions published in 2017 indicated that ONU with transurethral resection of the intramural ureter up to the extravesical fat followed by ureteral extraction is an oncologically safe and non-inferior approach (44).

\section{Intussusception technique for BCE}

Intussusception is a technique in which the ureter is transected, potentially leaving a portion of the intramural tunnel and the ureteral orifice behind (25). Thus, this approach is contraindicated in ureteral tumors and is reserved for patients with low-grade renal pelvic tumors $(25,41,44)$. Comparing the "pluck" technique with the ureteral stripping procedure, Geavlete et al. claimed that there are no differences between the techniques with regard to operative time, complications, and oncologic outcomes (45). However, this technique was associated with approximately $10 \%$ intraoperative complication rate with associated difficult $\mathrm{BCE}$, leading to open conversion in $9.5-12.5 \%$ in patients (45). When comparing ureteral stripping with open BCE, Saika et al. reported a significantly higher risk of intravesical recurrence with endoscopic ureteral stripping compared with the standard ONU with a designated incision for the distal ureterectomy and intramural bladder cuff (46).

\section{$L N U$ for $B C E$}

Benefits of pure laparoscopic dissection of the bladder cuff include shorter operative times and a shorter length of stay $(24,26)$. Moreover, laparoscopic extravesical stapling of the distal ureter avoids a large anterior cystotomy. This approach, however, risks leaving behind viable ureteral mucosa. The oncologic consequences are twofold: (I) the stapled margin cannot be assessed histologically, and (II) viable urothelium may be left behind, posing oncologic risks $(2,7)$.

Several studies that compared the various methods to distal ureteral excision suggest a higher incidence of positive surgical margins and local recurrence in the group of patients who underwent the laparoscopic stapling approach $(14,16,21,24,41,47,48)$. Specifically, Xylinas et al. found that laparoscopic extravesical stapling of the distal ureter and bladder cuff was associated with a higher incidence of positive surgical margins and local recurrence (21). Nevertheless, Ribal et al. reported in a recent meta-analysis that after LNU, the rates of bladder recurrence ranged from $19-43 \%$, and is similar to those observed in the ONU series (48). Ritch et al. retrospectively compared ONU to extravesical stapling with LNU to total laparoscopic dissection with results suggesting that laparoscopic extravesical stapling had cystoscopically appreciable remnants of the resected ureteral orifice in as many as $50 \%$ of cases when compared to none when the other, more definitive, approaches were employed (16).

When LNU was initially introduced, there was also a theoretical risk of port-site seeding; however, this risk appears to be quite small, with only a handful of case reports documenting this complication (41). Meanwhile, LNU extravesical approach that employs stapling may leave a staple line exposed to urine, serving as a nidus for infection and stone formation. These risks remain theoretical as long-term reports analyzing the lithogenic complications of exposed staple lines from LNUBCE are lacking (29). However, one report reported stone formation in $5.7 \%$ of their cohort at 20 months post-operatively (29).

\section{LNU with transvesical technique for BCE}

The transvesical approach aims to potentiate the dissection of the intramural tunnel by placing additional ports into the bladder. In a seminal manuscript, Tsivian et al. reported on the placement of two additional trocars in the ipsilateral lower abdomen after a standard LNU (49). With the assistance of these transvesical trocars, the surgeon performs the caudal ureteral dissection down to detrusor fibers at the UVJ prior to excising the bladder cuff with a laparoscopic device. Gill et al. modified the initial technique and reported on the transurethral technique assisted by two ports placed transvesically that allowed for earlier occlusion of the ureter, suggesting it may be oncologically safer than the pure transurethral approaches (14). They also compared their technique with the laparoscopic stapling approach and reported increased rates of positive margins, bladder recurrences, and distant metastasis in the stapling group, but did this finding did not achieve statistical significance $(40,50)$. 
Numerous variations of this transvesical technique have been reported with many techniques employing the pneumovesicum strategy (25,42-45). Despite initial enthusiasm, the approach appears to have fallen largely out favor due to the disadvantages of the need for repositioning, increased operative time, and, more importantly, the theoretical risk of trocar seeding and urine extravasation as the intravesical port sites are managed by prolonged bladder decompression with an indwelling catheter and are generally not closed primarily (30).

\section{RAL-NU technique for BCE}

Tsivian et al. initially reported in 2011 the first experience with pure RNU-BCE in 65 patients (49). Intermediate-term outcomes were published in 2015 and confirmed clinically comparable outcomes of RNU-BCE when compared to ONU and LNU approaches to BCE (51). Similar data was later reported by Lee et al. (34).

A meta-analysis of 7 RNU-BCE studies indicated favorable mean operative time, intraoperative blood loss, and duration of hospitalization when compared to nonrobotic approaches (15). In retrospective comparisons of pure ONU, LNU, and RAL-NU, the mean operative time of LNU appears to be longest at 164.8 to $462 \mathrm{~min}$, compared to ONU and RAL-NU at 156.2 to $324 \mathrm{~min}$ in these series (15). Furthermore, the meta-analysis reported that reported blood loss was highest in the ONU group, ranging from 300-750 mL, LNU group ranging from 144$580 \mathrm{~mL}$, and $75-270 \mathrm{~mL}$ for RNU (41). With regard to the length of stay, most contemporary series of RNU-BCE reported a median length of stay ranging from 3-4 days, somewhat shorter than was reported in previous years for LNU and ONU (41). Of course, such data with regard to all outcomes must be interpreted with extreme caution given their non-randomized and retrospective nature. It is difficult to believe that LNU and RNU result in significantly different outcomes in experienced hands. However, the costs of RNU are generally substantially higher and are of concern in the current healthcare environment (41).

While reports from robotic centers suggest non-inferior peri-operative outcomes when compared to LNU or ONU, the marked cost discrepancy between the laparoscopic and robotic approaches raises concerns regarding the value proposition of RAL-NU over LNU. In a recent study, the utilization of the robotic system incurred a significantly higher cost during the hospitalization, with estimated costs of $\$ 23,235$ vs. $\$ 17,637$ for RAL-NU and LNU, respectively (47).
Furthermore, the data assessing cost-effectiveness, value, and safety of the robotic approach in regard to distal ureteral tumors are lacking. This absence of safety and efficacy data are particularly salient in light of the recent FDA safety communication of February 28, 2019 (51). In this communication, the FDA reports concerns that health care providers and patients may not be fully aware of the safety and effectiveness of certain robotics applications in oncologic cases. Regulators cited that historically, the FDA's evaluation of the use of robotics has been focused on comparing complication rates at 30 days (51). In sum, they advise caution when utilizing robotic approaches to malignancies where efficacy and safety data are less robust.

Ultimately, RAL-NU must be performed by experienced providers. The currently available literature on pure RAL$\mathrm{NU}$ is based on retrospective series with short follow-up durations. The current European Association of Urology guidelines support the minimally invasive approach as an acceptable alternative for UTUC surgical management (3); however, the American Urological Association has not yet published organizational guidelines accepting a new gold standard approach.

\section{Evaluation of systemic chemotherapy}

Despite the fact that muscle-invasive UTUC has a worse stage-for-stage survival than muscle-invasive bladder cancer (MIBC), the role of neoadjuvant and adjuvant chemotherapy in UTUC is only now crystallizing. EAU and AUA guidelines for MIBC recommend neoadjuvant, cisplatin-based combined chemotherapy (3). However, data in the UTUC space have been limited by low patient enrollment and conflicting results. As a result, EAU and AUA Guidelines on UTUC indicate that the role of chemotherapy is reserved for metastatic disease as its efficacy in node-positive disease has yet to be established (3).

Most recently, robust data regarding the benefits of perioperative chemotherapy for patients with UTUC have started to emerge (52). Birtle et al. published preliminary results in 2018 from a phase III randomized trial of perioperative chemotherapy versus surveillance in UTUC or POUT trial assessing the use of adjuvant UTUC (52). This randomized controlled trial was conducted in the UK with two armed cohorts undergoing surveillance or adjuvant cisplatin-gemcitabine or carboplatin-gemcitabine chemotherapy after RNU for high-risk UTUC (52). Recruitment was terminated due to an early stopping rule for efficacy favoring the chemotherapy arm and thus acting 
as a landmark study supporting adjuvant chemotherapy as a new standard of care (52). Since eGFR often drops markedly following nephroureterectomy and many patients are then unable to benefit from cisplatin administration, receipt of neoadjuvant therapy is the preferred approach at many tertiary centers (53).

\section{Conclusions}

Retrospective comparisons of transvesical, extravesical, and endoscopic bladder cuff resection are of questionable generalizable validity due to the limitations of appropriately balancing measured and unmeasured confounders in preselected cohorts of patients. Some authors have demonstrated marginally higher rates of intravesical recurrence in patients with endoscopic resection without inferiority in extravesical recurrence nor survival $(13,15,20,38)$. Other authors have concluded that endoscopic transurethral resection is an oncologically safe strategy with notably shorter operative time, short hospitalization, and similar RFS and DSM to patients who undergo open bladder cuff resection $(7,10,36,45)$.

In summary, given the numerous surgical options and the absence of robust evidence demonstrating one approach as being superior to another, the urologic surgeon should use clinical judgment. The choice of approach must be nuanced and take into account each patient's disease, deliverables of each strategy, and one's surgical comfort level.

\section{Acknowledgments}

Funding: None.

\section{Footnote}

Provenance and Peer Review: This article was commissioned by the Guest Editors (John J. Knoedler and Jay D. Raman) for the series "Upper-Tract Urothelial Carcinoma: Current State and Future Directions" published in Translational Andrology and Urology. The article was sent for external peer review organized by the Guest Editors and the editorial office.

Conflicts of Interest: All authors have completed the ICMJE uniform disclosure form (available at http:// dx.doi.org/10.21037/tau.2020.01.17). The series "UpperTract Urothelial Carcinoma: Current State and Future Directions" was commissioned by the editorial office without any funding or sponsorship. The authors have no other conflicts of interest to declare.

Ethical Statement: The authors are accountable for all aspects of the work in ensuring that questions related to the accuracy or integrity of any part of the work are appropriately investigated and resolved.

Open Access Statement: This is an Open Access article distributed in accordance with the Creative Commons Attribution-NonCommercial-NoDerivs 4.0 International License (CC BY-NC-ND 4.0), which permits the noncommercial replication and distribution of the article with the strict proviso that no changes or edits are made and the original work is properly cited (including links to both the formal publication through the relevant DOI and the license). See: https://creativecommons.org/licenses/by-nc-nd/4.0/.

\section{References}

1. Xylinas E, Rink M, Cha EK, et al. Impact of Distal Ureter Management on Oncologic Outcomes Following Radical Nephroureterectomy for Upper Tract Urothelial Carcinoma. Eur Urol 2014;65:210-7.

2. Lee SM, McKay A, Grimes N, et al. Distal Ureter Management during Nephroureterectomy: Evidence form a Systemic Review and Cumulative Analysis. J Endourol 2019;33:263-273.

3. Roupret M, Babjunk M. European Association of Urology Guidelines on Upper Urinary Tract Urothelial Carcinoma: 2017 Update. Eur Urol 2018;73:111-22.

4. Adamo M, Dickie L, Ruhl J. SEER Program Coding and Staging Manual 2015. Bethesda, MD: National Cancer Institute, 2015.

5. Wein AJ, Kavoussi LR, Campbell MF, et al. CampbellWalsh Urology. PA, USA: Elsevier-Saunders, 2012.

6. Leow JJ, Chong KT, Chang SL, et al. Upper tract urothelial carcinoma: A different disease entity in terms of management. ESMO Open 2017;1:1-4.

7. Krabbe LM, Westerman ME, Bagrodia A, et al. Surgical management of the distal ureter during radical nephroureterectomy is an independent predictor of oncological outcomes: Results of a current series and review of the literature. Urol Oncol 2014;32:19-26.

8. Fiuk, JV, Schwartz BF. Upper tract urothelial carcinoma: Paradigm shift towards nephron sparing management. World J Nephrol 2016;5:158.

9. Li CC, Chang TH, Wu WJ, et al. Significant predictive 
factors for prognosis of primary upper urinary tract cancer after radical nephroureterectomy in Taiwanese patients.

Eur Urol 2008;54:1127-34.

10. Luo HL, Kang CH, Chen YC, et al. Oncological impact of endoscopic bladder cuff management during nephroureterectomy varies according to upper urinary tract tumor location. Int J Urol 2014;21:366-9.

11. Simhan J, Smaldone MC, Egleston BL, et al. Nephronsparing management vs radical nephroureterectomy for low- or moderate-grade, low-stage upper tract urothelial carcinoma. BJU International 2014;114:216-20.

12. Coleman JA, Wong NC, Sjoberg DD, et al. LBA17: Late-Breaking Abstract: Multicenter Prospective Phase II Clinical Trial of Gemcitabine and Cisplatin as Neoadjuvant Chemotherapy in Patients with High-Grade Upper Tract Urothelial Carcinoma. Chicago, IL: Annual Meeting of American Urological Association, 2019.

13. Hsueh TY, Huang YH, Chiu AW, et al. A comparison of the clinical outcome between open and hand-assisted laparoscopic nephroureterectomy for upper urinary tract transitional cell carcinoma. BJU International 2004;94(6):798-801.

14. Gill IS, Soble JJ, Miller SD, et al. A novel technique for management of the en bloc bladder cuff and distal ureter during laparoscopic nephroureterectomy. J Urol 1999;161:430-4

15. Allard CB, Alamri A. The method of bladder cuff excision during laparoscopic radical nephroureterectomy does not affect oncologic outcomes in upper tract urothelial carcinoma. World J Urol 2013;31:175-81.

16. Ritch CR, Kearns JT, Mues AC, et al. Comparison of distal ureteral management strategies during laparoscopic nephroureterectomy. J Endourol 2011;25:1149-54.

17. Darwiche F, Swain S, Kallingal G. et al. Operative technique and early experience for robotic-assisted laparoscopic nephroureterectomy (RALNU) using da Vinci Xi. Springer Plus 2015;4:298.

18. Rose K, Khan S, Godbole H, et al. GUY'S and St. Thomas' Robotics Group Robotic-assisted retroperitoneoscopic nephroureterectomy. Int J Clin Pract 2006;60:12-4.

19. Kapoor A, Dason S, Allard CB, et al. The impact of method of distal ureter management during radical nephroureterectomy on tumour recurrence. Can Urol Assoc J 2014;8:845-52.

20. Ko R, Chew RH, Hickling DR, et al. Transitional-cell carcinoma recurrence rate after nephroureterectomy in patients who undergo open excision of bladder cuff $\mathrm{v}$ transurethral incision of the ureteral orifice. J Endourol 2007;21:730-4.

21. Xylinas E, Colin P, Audenet F, et al. Intravesical recurrence after radical nephroureterectomy for upper tract urothelial carcinomas: predictors and impact on subsequent oncological outcomes from a national multicenter study. World J Urol 2013;31:61-8.

22. Lughezzani G, Sun M, Perrotte P, et al. Should bladder cuff excision remain the standard of care at nephroureterectomy in patients with urothelial carcinoma of the renal pelvis? A population-based study. International Braz J Urol 2010;36:641-2.

23. Braun AE, Uzzo RG, Kutikov A. Choosing the optimal strategy for bladder cuff management at nephroureterectomy. AUA News. Available online: https:// epub.auanet.org/publication/? $\mathrm{m}=52864 \& \mathrm{i}=587124 \& \mathrm{p}=6$

24. Stravodimos KG, Komninos C, Kural AR, et al. Distal ureterectomy techniques in laparoscopic and robotassisted nephroureterectomy: updated review. Urol Ann 2015;7:8-16.

25. Clayman RV, Garske GL, Lange PH. Total nephroureterectomy with ureteral intussusception and transurethral ureteral detachment and pull-through. Urology 1983;21:482-6.

26. Keeley FX, Sharma NK, Tolley D. A.: Hand-assisted laparoscopic nephroureterectomy. BJU Int 1999;83:504.

27. Shariat SF, Zigeuner RE, Wood G, et al. Proposal for Sublcassification of pT3 Urothelial Cancer of the Renal Pelvis. J Urol 2009;181:133-4.

28. Guo G, Yang Y, Dong J, et al. A new 2-micrometer continuous wave laser method for management of the distal ureter in retroperitoneal laparoscopic nephroureterectomy. J Endourol 2015;29:430-4.

29. Mueller TJ, DaJusta DG, Cha DY, et al. Ureteral fibrin sealant injection of the distal ureter during laparoscopic nephroureterectomy - a novel and simple modification of the pluck technique. Urology 2010;75:187-92.

30. Zou X, Zhang G, Wang X, et al. A one-port pneumovesicum method in en bloc laparoscopic nephroureterectomy with bladder cuff resection is feasible and safe for upper tract transitional cell carcinoma. BJU Int 2011;108:1497-500.

31. Ou CH, Yang WH, Tzai TS, et al. A modified supine position to speed hand assisted retroperitoneoscopic nephroureterectomy: the Johnnie Walker position. J Urol 2006;176:2063-7.

32. Nanigian DK, Smith W, Ellison LM. Robot-assisted laparoscopic nephroureterectomy. J Endourol 
2006;20:463-5.

33. Eandi JA, Nelson RA, Wilson TG, et al. Oncologic outcomes for complete robot-assisted laparoscopic management of upper-tract transitional cell carcinoma. J Endourol 2010;24:969-75.

34. Lee Z, Cadillo-Chavez R, Lee DI, et al. The technique of single stage pure robotic nephroureterectomy. J Endourol 2013;27:189-95.

35. Li WM, Shen JT, Li CC, et al. Oncologic outcomes following three different approaches to the distal ureter and bladder cuff in nephroureterectomy for primary upper urinary tract urothelial carcinoma. Eur Urol 2010;57:963-9.

36. Walton TJ, Sherwood BT, Parkinson RJ, et al. Endoscopic Ureteral Detachment and Formal Bladder Cuff Excision in Open Nephroureterectomy for Upper Urinary Tract Transitional Cell Carcinoma. J Urol 2009;181:532-9.

37. Toussi A, Miest, T, Boorjian, S, et al. Oncological Outcomes Comparing Intravesical and Extravesical Bladder Cuff Excision Following Radical Nephroureterectomy for Upper Tract Urothelial Carcinoma. J Urol 2017;197:1275-6.

38. Seisen T, Granger B, Colin P, et al. A systematic review and meta-analysis of clinicopathologic factors linked to intravesical recurrence after radical nephroureterectomy to treat upper tract urothelial carcinoma. Eur Urol 2015;67:1122-33.

39. Lin WC, Hu FC, Chung SD, et al. The role of lymphovascular invasion in predicting the prog-nosis of clinically localized upper tract urothelial carcinoma (pT13cN0M0). J Urol 2008;180:879.

40. Srirangam SJ, Van Cleynenbreugel B, Van Poppel H. Laparoscopic nephroureterectomy: The distal ureteral dilemma. Adv Urol 2009. doi: 10.1155/2009/316807.

41. Rai BP, Shelley M, Coles B, et al. Surgical management for upper urinary tract transitional cell carcinoma (UUTTCC): a systematic review. BJU Int 2012;110:1426-35.

42. Cormio L, Selvaggio O, Di Fino G, et al. Transurethral distal ureter balloon occlusion and detachment: a simple means of managing the distal ureter during radical nephroureterectomy. J Endourol 2013;27:139-42.

43. Agarwal DK, Khaira HS, Clarke D, et al. Modified transurethral technique for the management of distal ureter during laparoscopic assisted nephroureterectomy. Urology 2008;71:740-3.

44. Fragkoulis C, Pappas A, Papadopoulos G, et al.
Transurethral resection versus open bladder cuff excision in patients undergoing nephroureterectomy for upper urinary tract carcinoma: Operative and oncological results. Arab J Urol 2017;15:64-7.

45. Geavlete P, Georgescu D, Bancu S, et al. Endoscopic ureteral procedures for one-step nephroureterectomy: experience in 100 cases. J Endourol2007;21:1019-24.

46. Saika T, Nishiguchi J, Tsushima T, et al. Comparative study of ureteral stripping versus open ureterectomy for nephroureterec- tomy in patients with transitional carcinoma of the renal pelvis. Urology 2004;63:848-52.

47. Trudeau V, Gandaglia G. Robot-assisted versus laparoscopic nephroureterectomy for upper tract urothelial cancer: A population-based assessment of costs and preoperative outcomes. Can Urol Assoc J 2014;8:695-701.

48. Ribal MJ, Huguet J, Alcaraz A. Oncologic outcomes obtained after laparoscopic, robotic and/or single port nephroureterectomy for upper urinary tract tumours. World J Urol 2013;31:93-107.

49. Tsivian A, Benjamin S, Sidi AA. A sealed laparoscopic nephroureterectomy: A new technique. Eur Urol 2007;52:1015-9.

50. Aboumohamed AA, Krane LS, Hemal AK. Oncologic Outcomes Following Robot-Assisted Laparoscopic Nephroureterectomy with Bladder Cuff Excision for Upper Tract Urothelial Carcinoma. J Urol 2015;194:1561-6.

51. U.S. Department of Health and Human Services. U.S. Food and Drug Administration. Federal Caution When Using Robotically-Assisted Surgical Devices in Women's Health including Mastectomy and Other Cancer-Related Surgeries: FDA Safety Communication, 2019.

52. Birtle A, Catto J, Johnson M, et al. Results of Pout - a Phase III Randomised Trial of Peri-Operative Chemotherapy Versus Surveillance in Upper Tract Urothelial Cancer (Utuc). J Urol 2018;199:213-4.

53. Lane BR, Smith AK, Larson BT, et al. Chronic kidney disease after nephroureterectomy for upper tract urothelial carcinoma and implications for the administration of perioperative chemotherapy. Cancer 2010;116:2967-73.

Cite this article as: Braun AE, Srivastava A, Maffucci F, Kutikov A. Controversies in management of the bladder cuff at nephroureterectomy. Transl Androl Urol 2020;9(4):1868-1880. doi: 10.21037/tau.2020.01.17 